Matthias RIEDEL, Giuseppe Fabrizio TURRISI

\title{
Contribution to the knowledge of Ichneumonidae from Sicily. II. Ichneumoninae (Hymenoptera)
}

\begin{abstract}
Riassunto: Contributo alla conoscenza degli Ichneumonidae di Sicilia. II. Ichneumoninae (Hymenoptera). Sono forniti dati faunistici e tassonomici riguardanti gli Hymenoptera Ichneumonidae (Ichneumoninae) della Sicilia. Sono segnalate 79 specie, 39 delle quali sono nuove per la Sicilia e 9 anche per l'Italia. Il numero di specie di Ichneumoninae note per la Sicilia è elevato a 105 .
\end{abstract}

\begin{abstract}
Faunistic and taxonomic data on Hymenoptera Ichneumonidae (Ichneumoninae) from Sicily are provided. 79 species are recorded, 39 of which are new for Sicily and 9 are new also for Italy. The number of the species of Ichneumoninae known for Sicily is increased up to 105 .
\end{abstract}

Key words: Ichneumonidae, Ichneumoninae, new records, Italy, Sicily.

\section{INTRODUCTION}

With more than 24,000 valid extant species worldwide the Ichneumonidae is one of the largest families of the Hymenoptera (Yu et al., 2005). About 4,5005,000 species have been found in Europe so far. Therefore, this Hymenoptera family represents the most diverse insect group in most European countries. But despite their abundance and important role in ecosystems and as biological pest control, the taxonomy, ecology, and distribution of many groups of Ichneumonidae still remain incompletely known. The Ichneumoninae represents a very species-rich subfamily, usually parasitizing Lepidoptera, containing 392 species known for Italy, of which only 66 presently recorded for Sicily (Scaramozzino, 1995; Turrisi et al., 2007).

The faunistic knowledge of the Ichneumonidae from Sicily has received very little attention from researchers, and most part of data comes from old contributions, e.g. Ghiliani (1842), Sichel (1860), De Stefani Perez (1882, 1883, 1884, 1885, 1886, 1887a, b, 1894, 1895) and Riggio \& De Stefani, 1888. Only a few contributions were added recently: Pagliano \& Scaramozzino (1995), Pagliano (2003), both containing data from some circumsicilian islands, Turrisi et al. (2007), treating Ichneumoninae: Phaeogenini and Diplazontinae and Riedel (in Riedel \& Tomarchio, 2012a). The most recent survey of Sicilian Ichneumonidae still remains the checklist of the Italian Ichneumonidae (Scaramozzino, 1995), although mostly not containing original data on Sicilian fauna.

The present contribution deals with records of many species belonging to the subfamily Ichneumoninae, mostly being first records for Sicily and some for Italy. The results of this contribution have been previously presented by Riedel \& Tomarchio (2012a) and subsequently invalidated by the same authors (Riedel \& Tomarchio, 2012b), except the description of Platylabus rufator Riedel, 2012, which is valid and is incorporated in the faunistic species list.

This research belongs to a broader project for a better knowledge of faunistics, taxonomy, distribution, and biogeography of the Sicilian Ichneumonidae, primarily based on the study of the G.F. Turrisi collection.

\section{Materials AND Methods}

This study is based on examination of the following two collections, identified in the text with acronyms:

UCTC: University of Catania, Turrisi G.F. collection (Catania, Italy).

STC: Tomarchio S. collection (San Gregorio di Catania, Catania, Italy).

Specimens were usually collected by hand-netting, sometimes on flowers of several Angiospermae, especially in the eastern part of Sicily. A list of the collecting sites for the material belonging to the Turrisi collection was already provided by Turrisi et al. (2007), including brief descriptions of habitat. To avoid repetitions, the name of the collector is omitted if it is the same of the depository. Primary data on Sicilian Ichneumonidae were obtained from the papers by De Stefani Perez, especially the comprehensive 
catalogue of Sicilian Hymenoptera (De Stefani Perez, 1895) and the checklist of the Italian Ichneumonidae (Scaramozzino, 1995); distributional data were taken from the catalogues of the Ichneumonidae of the World by Yu \& Horstmann (1997) and Yu et al. (2005).

The identification of the material was made by the first author; we only excluded some specimens with uncertain determination, mainly some of the genus Ichneumon.

\section{Results: List of Species}

\section{Phaeogenini}

Aethecerus nitidus Wesmael, 1845

Material. Erei, Calascibetta (Enna), 1 ○े 1.XI.1994 (UCTC).

Distribution. Western Palaearctic region, known from Italy (Scaramozzino, 1995), new for Sicily.

Diadromus heteroneurus Holmgren, 1890

Material. Etna Ovest, Mt. Maletto, 1600 m, 1 o 20.VII.1999 (STC).

Distribution. Western Palaearctic region, new for Italy.

\section{Listrodromini}

Anisobas brombacheri Heinrich, 1933

Material. Etna, San Gregorio di Catania (CT) 200 m, 1ठ 4.v.1997 (UCTC).

Distribution. Western Palaearctic region, known from Sicily (Horstmann, 2007).

Anisobas cingulatellus Horstmann, 1997 syn. Anisobas cingulatorius (Gravenhorst, 1829) praeocc.

Material. Erei, Aidone (EN), 760 m, Case Bonincontro, 1 8.V.2000 (STC); Piana di Catania, Contrada

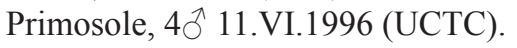

Distribution. Widespread in the Palaearctic region, known from Italy (Horstmann, 2007, Scaramozzino, 1995 as Anisobas cingulatorius), new for Sicily.

Anisobas rebellis Wesmael, 1845

Material. Etna, Linguaglossa, Mt. Baracca, 1700 m, 1 ㅇ 26.VIII.1994 (UCTC); Fiume Vizzini, Vizzini (CT), Poggio del Lago 600 m, 1§̂ 7.V.1994 (UCTC).
Distribution. Widespread in the Palaearctic region, known from Sicily (Horstmann, 2007).

Listrodromus nycthemerus (Gravenhorst, 1820)

Material. Etna, Zafferana Etnea), Valle San Giacomo (CT, 800 m, 1 ㅇ 12.VII.1995 (UCTC).

Distribution. Widespread in the Palaearctic region, known from Italy (Scaramozzino, 1995), new for Sicily.

\section{Platylabini}

Apaeleticus bellicosus Wesmael, 1845

Material. Etna, Mt. Rossi (Nicolosi, CT), 750 m, 1 ô 1.V.1994 (UCTC); Nebrodi, Foresta di Malabotta, Roccella Valdemone (ME), 1000 m, 1 ð 28.VIII.1995 (UCTC).

Distribution. Western Palaearctic region, known from Italy (Scaramozzino, 1995), new for Sicily.

Apaeleticus inimicus (Gravenhorst, 1820)

Material. Piana di Catania, Contrada Primosole, 10 20.IV.1997 (UCTC).

DistRIBUTION. Western Palaearctic region, known from Italy (Scaramozzino, 1995), new for Sicily.

Apaeleticus mesostictus (Gravenhorst, 1829)

Material. Piana di Catania, Contrada Primosole, $1 \hat{\sigma}$ 2.V.1998 (UCTC).

Distribution. Palaearctic region, new for Italy.

Cyclolabus pactor (Wesmael, 1845)

Material. Etna, Tremestieri Etneo (Catania), $350 \mathrm{~m}$,

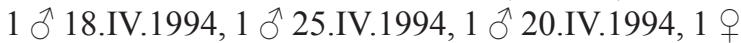
16.V.1994, 1 ○ 20.v.1995, 1 ㅇ 28.IV.1996 (UCTC); Etna, Mt. Rossi (Nicolosi, CT) 750 m, 1 ô 1.v.1994 (UCTC); Etna, San Gregorio di Catania (CT), $300 \mathrm{~m}$, 2 กै 20.IV.1994, 1 đึ 4.V.1997 (UCTC).

Distribution. Western Palaearctic region, known from Italy (Scaramozzino, 1995), new for Sicily.

Linycus exhortator (Fabricius, 1787)

Material. Etna, Tremestieri Etneo (CT), 1 o 22.III.1994 (UCTC).

Distribution. Widespread in the Holarctic region, known from North Italy (Pagliano, 2009) and Sicily (Scaramozzino, 1995). 
Platylabus iridipennis (Gravenhorst, 1829)

Material. Etna, dintorni Mt. Rossi (Nicolosi, CT), 700 m, 1 ठै 12.X.1997 (UCTC).

Distribution. Western Palaearctic region, known from Italy (Riedel, 2008), new for Sicily.

Platylabus obator (Desvignes, 1856)

Material. Etna, Tremestieri Etneo (CT), 1 ๙ 20.III.1994 (UCTC); Etna, Maletto, Contrada Fontanamurata (CT), 1000 m, 2 ô 18.V.1996 (UCTC).

Distribution. Western Palaearctic region, known from Italy (Roberti et al., 1965), new for Sicily.

\section{Platylabus rufator Riedel, 2012}

Material. Etna, Tremestieri Etneo (CT) 350 m, 1 29.III.1993 (UCTC).

Distribution. Recently described (Riedel \& Tomarchio, 2012a) and so far only known from Sicily.

Platylabus tricingulatus (Gravenhorst, 1820)

Material. Etna, Milo (CT), 750 m, 1 21 .VII.1998 (UCTC).

Distribution. Western Palaearctic region, known from Italy (Scaramozzino, 1995, Riedel 2008), new for Sicily.

\section{Joppocryptini}

Pseudoplatylabus uniguttatus (Gravenhorst, 1829)

Material. Etna,Tremestieri Etneo (CT), 350 m, 1 o 9.XI.1994 (UCTC).

Distribution. Western Palaearctic region, new for Italy.

\section{Trogini}

Trogus lapidator (Fabricius, 1787)

Material. Iblei, Fiume Vizzini, Vizzini (CT), 1 q 31.V.1992 (UCTC).

Distribution. Holarctic and Oriental regions, known from North Italy (Pagliano, 2009) and Sicily (Scaramozzino, 1995).

\section{Heresiarchini}

Coelichneumon biguttorius (Thunberg, 1789) syn. serenus Gravenhorst

Material. Iblei, Buccheri (CT), 2 ઈ 24.V.2000
(STC); Etna, Milo, Foresta Cubania (CT), 1400 m, 1 ○ 6.IX.1995, Alicata \& Giusso leg. (UCTC).

Distribution. Western Palaearctic region, known from Sicily (Rudow, 1888, Horstmann, 1993, 2002).

Coelichneumon erythromerus (Rudow, 1888)

Material. Etna, Ragalna, Contrada Milia (CT), 1500 m, 1 ô 30.VIII.1992 (STC).

Distribution. Western Palaearctic region, known from North Italy (Pagliano, 2009) and Sicily (Scaramozzino, 1995).

Coelichneumon graecus Horstmann, 2002

Material. Iblei, Pachino, Pantano Longarini (SR), , 1 ô 4.VI.1995, S. Bella leg. (UCTC).

DistRIBUTION. previously only known from Greece, new for Italy.

Coelichneumon oltenensis Constantineanu, Pirvescu \& Mihalache, 1979

Material. Selinunte (TP), 2 ○ 13.IV.1965 (Natural History Museum, London).

DistRibution. previously known from only Great Britain and Romania, new for Italy.

Coelichneumon quadriannulatus (Gravenhorst, 1829)

Material. Etna, Milo, Casa Pietracannone (CT) 1100 m, 1 กิ 12.VII.1998 (UCTC); Etna, Maletto, Contrada Fontanamurata (CT), $1000 \mathrm{~m}, 1$ กे 29.VI.1996, 3 ठै 7.VII.1996 (UCTC).

Distribution. previously known from France, Germany, and Poland, new for Italy.

Protichneumon fusorius fusorius (Linnaeus, 1761)

Material. Etna, Linguaglossa, Mt. Baracca (CT), 1700 m, 1 กै 8.VIII.1996 (UCTC).

Distribution. Palaearctic region, known from North Italy (Pagliano, 2009) and Sicily (Sichel, 1860).

Syspasis carinator (Fabricius, 1798)

Material. Etna, Milo, Fornazzo (CT), 800 m, 1 o 8.X.1994 (UCTC).

Distribution. Western Palaearctic region, known from Italy (Scaramozzino, 1995 as helleri), new for Sicily. 
Syspasis leucolomia (Gravenhorst, 1829)

Material. Etna, Maletto, Contrada Fontanamurata (CT), 1000 m, 1 §ै 26.X.1997 (UCTC).

Distribution. Western Palaearctic region, known from Italy (Pagliano, 2009) and Sardinia (Pic, 1899), new for Sicily.

Syspasis scutellator (Gravenhorst, 1829)

MATERIAl. Etna, Linguaglossa, Mt. Baracca (CT), 1700 m, 2 ô 23.VIII.1995 (UCTC); Etna, Maletto, Contrada Fontanamurata (CT), $1000 \mathrm{~m}, 1 \hat{\jmath}$ 7.VII.1996 (UCTC).

Distribution. Widespread in the Palaearctic region, known from Italy (Pagliano, 2009) and Sicily (Scaramozzino, 1995).

\section{Ichneumonini}

Amblyteles armatorius Forster, 1771

Material. Etna, Contrada S. Leo, Belpasso (CT), $1100 \mathrm{~m}, 1$ ㅇ 29.V.1991 (UCTC); 1 ๙ 17.V.1992; 1 ภ 16.V.1994 (STC); Etna, San Gregorio di Catania (CT), 1 † 5.V.1994 (UCTC); Iblei, Buccheri (SR), 1 గ 24.V.2000 (STC).

Distribution. Widespread in the Palaearctic region, known from Sicily (Sichel, 1860; De Stefani Perez, 1896).

\section{Baranisobas ridibundus (Gravenhorst, 1829)}

Material. Etna, Ragalna, Mt. Vetore (CT), 1700 m, 2 กิ 31.VIII.1997; 3 ก 26.VIII.1995 (UCTC); Etna, Milo, Casa Paternò (CT), $1300 \mathrm{~m}$ (CT), 1 ઈ 29.VIII.1996 (UCTC); Iblei, Fiume Vizzini, Contrada Rubalà (CT), 320 m, 1 đ 1.X.1996 (UCTC); Nebrodi, Roccella Valdemone, Foresta di Malabotta, (ME) 1000 m, 1 đ 28.VIII.1995 (UCTC); Catania, San Nullo, 1 o 4.VI.1994 (UCTC); Etna, Tremestieri Etneo (CT), 350 m, 1 đ̊ 9.XI.1994 (UCTC); Etna, Milo Fornazzo (CT), 800 m, 1 15.IX.1994 (UCTC).

Distribution. Western Palaearctic region, known from Italy (Scaramozzino, 1995), new for Sicily.

Barichneumon bilunulatus (Gravenhorst, 1829)

Material. Etna, Milo, Casa Pietracannone (CT), 1100 m, 1 ठ 12.VII.1998 (UCTC).

Distribution. Widespread in the Palaearctic region, known from Italy (Pagliano, 2009) and Sicily (De Stefani Perez, 1895; Scaramozzino, 1995).
TAXONOMICAL REMARK. The available $\delta$ has an ivory stripe on the flagellomeres 15-17 (var. subannulatus Berthoumieu).

Barichneumon derogator (Wesmael, 1845)

Material. Etna, Tremestieri Etneo (CT), 350 m, 2 đ 1.VI.1994, 2 § 7.VI.1994 (UCTC).

Distribution. Widespread in the Palaearctic region, known from Sicily (De Stefani Perez, 1895, Scaramozzino, 1995).

Barichneumon gemellus (Gravenhorst, 1829)

Material. Etna, Milo (CT), 750 m, 1 đ̊ 21.VII.1998 (UCTC).

Distribution. Widespread in the Palaearctic region, new record for Italy.

Barichneumon lituratae (Hartig, 1838)

Material. Etna, Ragalna, Contrada Milia (CT), 1250 m, 1 ठิ 9.VI.1996 (UCTC); Etna, Maletto, Contrada Fontanamurata (CT), $1000 \mathrm{~m}, 2$ ठे 9.VII.1995 (UCTC); Etna, Belpasso, Mt. S. Leo (CT), 1000 m, 1 ơ 4.VII.1992 (UCTC).

Distribution. Western Palaearctic region, new for Italy.

Barichneumon sedulus (Gravenhorst, 1820)

Material. Etna, Maletto, Contrada Fontanamurata (CT), 1000 m, 1 đ 28.VI.1998 (UCTC); Etna, Ragalna, Serra La Nave (CT), 1730 m, 1 đ̊ 26.VI.1999 (UCTC); Paternò, Contrada Petulenti (CT), 1 đ 3.VI.1993 (STC); Iblei, Vizzini (CT), 1 ðิ 21.V.2000 (STC); Etna, Milo, Casa Pietracannone (CT), 1100 m, 1 đ 12.VII.1998 (UCTC); Madonie, Petralia Sottana, Piano Battaglia, Valle di Zotta Fonda (PA), 1650 m, 1 ठ̊ 14.VII.1993 (UCTC).

Distribution. Widespread in the Palaearctic region, known from Sicily (De Stefani, 1894).

\section{Cratichneumon coruscator (Linnaeus, 1758)}

Material. Etna, Linguaglossa, Mt. Baracca (CT), 1700 m, 1 ô 21.VIII.1996 (UCTC); Etna, Ragalna, Contrada Milia (CT), 1400 m, 1 o 10.VIII.1999 (STC); Iblei, Fiume Vizzini, Contrada Rubalà (CT), 320 m, 1 \& 16.V.1999 (UCTC); Etna, Ragalna, Mt. Vetore (CT), 1750 m, 1 § 26.VIII.1995 (UCTC); Nebrodi, Roccella Valdemone, Foresta di Malabotta 
(ME), 1000 m, 1 ô 28.VIII.1995 (UCTC).

Distribution. Widespread in the Palaearctic region, known from Italy (Scaramozzino, 1995), new for Sicily.

\section{Cratichneumon culex (Müller, 1776)}

Material. Etna Ovest, Mt. Maletto, $1600 \mathrm{~m}, 1$ o 20.VIII.1999 (STC).

Distribution. Widespread in the Palaearctic region, known from Italy (Scaramozzino, 1995), known from Sicily (Cecconi, 1924).

\section{Cratichneumon viator (Scopoli, 1763)}

Material. Nebrodi, Roccella Valdemone, Foresta di Malabotta (ME), 1000 m, 1 ○े 28.VIII.1995 (UCTC); Etna, Mt. Maletto (CT), 1600 m, 1 ภ 20.VII.1999 (STC); Etna, Milo, Mt. Scorsone (CT) 1600 m, 1 oิ 10.VIII.1996 (UCTC); Etna, Milo, Casa Pietracannone (CT), 1100 m, 1 ○े 28.VIII.1996 (UCTC); Etna, Ragalna, Mt. Vetore (CT), 1700 m () 1 § 31.VIII.1997 (UCTC); Etna, Castiglione di Sicilia Caserma Pitarrone (CT), $1450 \mathrm{~m} \mathrm{(),} 1$ 30.II.1998 (UCTC).

Distribution. Widespread in the Holarctic Realm, known from Italy and Sardinia (Scaramozzino, 1995), new for Sicily.

\section{Crypteffigies pseudocryptus (Wesmael, 1857)}

Material. Etna, Linguaglossa, Mt. Baracca (CT), 1700 m, 1 ๙ 7.VII.1995, 2 ○े 23.VIII.1995 (UCTC).

Distribution. Palaearctic region, known from Italy (Scaramozzino, 1995), new for Sicily.

Crytea erythraea (Gravenhorst, 1820)

Material. Etna, Milo (CT), 750 m, 1 §ิ 21.VII.1998 (UCTC).

Distribution. Western Palaearctic region, known from Italy (Scaramozzino, 1995), new for Sicily.

Ctenichneumon divisorius (Gravenhorst, 1820)

Material. Etna, Tremestieri Etneo (CT), 350 m, 1 q 24.III.1994 (UCTC).

Distribution. Palaearctic region, known from Italy (Scaramozzino, 1995), new for Sicily.

Ctenichneumon melanocastaneus (Gravenhorst, 1820) Material. Etna, Tremestieri Etneo (CT), 350 m, 1 o
9.III.1994; 2 ○ 20.III.1994; 1 ㅇ 22.III.1994; 1 우 24.III.1994 (UCTC); Etna, Monterosso Etneo (CT), $500 \mathrm{~m}, 1$ ㅇ 21.IV.1996 (UCTC).

Distribution. Palaearctic region, known from Italy (Scaramozzino, 1995), new for Sicily.

Ctenichneumon messorius (Gravenhorst, 1820)

Material. Etna, Maletto, Contrada Fontanamurata (CT), $1000 \mathrm{~m}, 1$ q 29.VI.1996, 1 q 7.VII.1996 (UCTC); 1 \& IV. 1999, A. Messina leg. (UCTC).

Distribution. Palaearctic region, known from Italy (Pagliano, 2009) and Sicily (De Stefani Perez, 1895).

Ctenichneumon repentinus (Gravenhorst, 1820)

Material. Etna, Maletto, Contrada Fontanamurata (CT), 1000 m, 1 ð 28.VI.1998 (UCTC).

DistRIBUtion. Palaearctic region, known from Italy (Pagliano, 2009) and Sicily (Rudow, 1888).

Ctenochares bicolorus (Linnaeus, 1767)

Material. Piana di Catania, Passo Martino $37^{\circ} 24^{\prime} \mathrm{N}$ $16^{\circ} 02^{`} \mathrm{E}, 1$ ○ 15.VI.2010 (STC); Catania, 1 우 7.III.1997 (STC) (2319), 1 ㅇ 14.V.1998 (STC); Iblei, Fiume Vizzini, Vizzini (CT), 1 16.IX.1993 (STC); Etna Ovest, Randazzo, Lago Gurrida (CT), 800 m, 1 o 18.VIII.2001 (STC); Piana di Catania Primosole, 2 ô 11.VI.1996 (UCTC); Etna, Zafferana Etnea, Valle S. Giacomo (CT), 750 m, 1 ð 21.VIII.1991 (UCTC); Iblei, Fiume Vizzini (CT), 320 m, 1 16.V.1999 (UCTC).

Distribution. Widespread in the Palaearctic, Ethiopian and Australasian regions, known from Sicily (De Stefani Perez, 1895) and Lampedusa (Pagliano \& Scaramozzino, 1995).

Diphyus amatorius (Müller, 1776)

Material. Etna, Belpasso, Mt. San Leo (CT), 1000 m, 1 § 25.V.2004 (STC).

Distribution. Widespread in the Palaearctic region and known from Italy (Scaramozzino, 1995), new for Sicily.

Diphyus castanopyga (Stephens, 1835)

Material. Nebrodi, Cesarò, Mt. Soro, Portella Femmina Morta (ME), $1520 \mathrm{~m}, 1$ o 19.VIII.1991 (UCTC); Etna, Maletto, Contrada Fontanamurata (CT), 1000 m, 1 §ै 7.VII.1996 (UCTC). 
Distribution. Western Palaearctic region and known from Italy (Pagliano, 2009).

Diphyus mercatorius mercatorius (Fabricius, 1793) Material. Etna, Maletto, Contrada Fontanamurata (CT), 1000 m, 1 đे 28.VI.1998 (UCTC); Etna, Ragalna, Contrada Milia (CT), 1250 m, 1 ô 2.VI.1993 (UCTC); Etna, Belpasso, Mt. San Leo (CT), 1000 m, 1 กิ 24.VI.1982 (UCTC).

Distribution. Palaearctic region, known from Italy (Scaramozzino, 1995), new for Sicily.

Diphyus quadripunctorius (Müller, 1776)

Material. Etna, Belpasso, Mt. San Leo (CT), 1000 m, 1 ○ 4.VI.1992 (UCTC); Etna, Tremestieri Etneo (CT), $350 \mathrm{~m}, 1$ ก 16.V.1994 (UCTC).

Distribution. Widespread in the Palaearctic and Oriental regions, known from Italy (Alma \& Arzone, 1994) and Sicily (Sichel, 1860).

Diphyus pseudomercator Heinrich, 1978

Material. Etna, Belpasso, Mt. San Leo (CT), 1000 m, 1 \& 16.V.1999 (STC).

Distribution. Western Palaearctic region, known from Italy (Scaramozzino, 1995), new for Sicily.

Diphyus trifasciatus (Gravenhorst, 1829)

Material. Nebrodi, Cesarò, Mt. Soro Portella Femmina Morta (ME), 1520 m, 1 ð 11.VIII.1991 (UCTC).

Distribution. Palaearctic region, known from Sicily (Scaramozzino, 1995).

Eupalamus oscillator Wesmael, 1845

Material. Etna Ovest, Pendici Mt. Maletto (CT), 1600 m, 1 q 28.VII.1999 (STC).

Distribution. Palaearctic region, known from Italy (Scaramozzino, 1995), new for Sicily.

\section{Eutanyacra glaucatoria (Fabricius, 1793)}

Material. Paternò (CT), Contrada Petulenti, 1 q 9.V.1993 (STC) (547), 1 q 9.V.1993 (STC); Etna, Belpasso, Mt. S. Leo (CT), 1000 m, 2 Ô 16.V.1999 (STC); Etna, Randazzo, La Gurrida (CT), 850 m, 1 22.VIII.1995 (UCTC).

Distribution. Widespread in the Palaearctic region, known from Italy (Pagliano, 2009) and Sicily (Sichel, 1860; De Stefani Perez, 1894).
Eutanyacra picta (Schrank, 1776)

Material. Etna, Tremestieri Etneo (CT), 350 m, 1 đิ 15.IV.1993; 1 ㅇ 2.VI.1998; 1 q 28.VI.1998 (UCTC); Etna, Piedimonte Etneo (CT) Contrada Terremonte, 1 o 1.VI.1993, ex Euxoa fritici (Linnaeus, 1761) (Lep., Noctuidae), G. Costa leg. (UCTC); Iblei, Portopalo di. C.P., Capo delle Correnti (SR), light trap, 13 ô and 3 § 31.VII.1992 (UCTC).

Distribution. Widespread in the Palaearctic and Oriental region, known from Italy (Scaramozzino, 1995), Sardinia and Sicily (Pagliano, 2009).

Homotherus locutor (Thunberg, 1824)

MATERIAl. Etna, Linguaglossa, Mt. Baracca, $1700 \mathrm{~m}$, 1 ภ 21.VIII.1996 (UCTC); Nebrodi, Roccella Valdemone, Foresta di Malabotta (ME), $1000 \mathrm{~m}, 1$ ก 24.VII.1994 (UCTC); Etna, San Gregorio di Catania (CT), 200 m, 1 đ̊ 20.IV.1994 (UCTC); Etna, Tremestieri Etneo (CT), 1 ô 9.III.1994, 2 ô 1.IV.1994 (UCTC); Etna, Maletto, Contrada Fontanamurata (CT), 1000 m, 1 §ิ 7.VII.1996 (UCTC).

Distribution. Palaearctic region, known from Italy (Scaramozzino, 1995), new for Sicily.

Hoplismenus axillatorius (Thunberg, 1824)

Material. Piana di Catania, Contrada Primosole, 1 + 8.VIII.1996 (UCTC).

Distribution. Palaearctic region, known from Sicily (Sichel, 1860, De Stefani Perez, 1895).

Ichneumon affector affector Tischbein, 1879

Material. Nebrodi, Cesarò, Biviere di Cesarò (ME), 1274 m, 1 23.VI.1999 (UCTC).

Distribution. Western Palaearctic region, known from Italy (Scaramozzino, 1995), new for Sicily.

TAXONOMICAL REMARK. The $q$ has the hind tibia completely black, fourth metasomal tergite red in the basal half, and pterostigma yellowish-brown, otherwise as described by Hilpert (1992).

Ichneumon balteatus Wesmael, 1845

Material. Etna, Maletto, Mt. Maletto (CT), 1550 m, 1 ठ 24.VII.1992 (STC) (151).

Distribution. Palaearctic region, known from Italy (Scaramozzino, 1995), new for Sicily. 


\section{Ichneumon cessator Müller, 1776}

Material. Nebrodi, Cesarò, Biviere di Cesarò (ME), 1274 m, 2 ○े 23.VI.1999 (UCTC); Etna, Linguaglossa, Mt. Baracca (CT), 1700 m, 2 กิ 8.VIII.1996, 1 ภ 21.VIII.1996 (UCTC).

Distribution. Palaearctic region, known from Italy (Scaramozzino, 1995), new for Sicily.

\section{Ichneumon extensorius Linnaeus, 1758}

MATERIAL. Etna, Milo, Mt. Scorsone 1600 m (CT), 3 đ 10.VIII.1996 (UCTC); Etna, Milo, Casa Pietracannone (CT), 1150 m, 1 § 8.VIII.1996 (UCTC); Etna, Linguaglossa, Mt. Baracca (CT), $1700 \mathrm{~m}, 3$ ๙ 8.VIII.1996, 2 § 21.VIII.1996 (UCTC); Etna, S. Alfio Pietrafucile (CT), 850 m, 1 đૈ 28.VII.1998 (UCTC); Etna, Castiglione di Sicilia, Foresta Ragabo (CT), 1500 m , 1 20.XII.1997, S. Bella leg. (UCTC); Etna, Milo, Foresta Cubania (CT), 1400 m, 1 6.IX.1995, Alicata \& Giusso leg. (UCTC); Nebrodi, Cesarò, Biviere di Cesarò (ME), 1274 m, 1 o 23.VI.1999 (UCTC).

Distribution. Palaearctic region and known from Italy (Pagliano, 2009) and Sicily (Scaramozzino, 1995).

\section{Ichneumon gracilicornis Gravenhorst, 1829}

Material. Etna, Bronte, Mt. Palestra (CT), 1900 m, 1 ㅇ 24.VIII.1996 (UCTC); Etna, Linguaglossa, Mt. Baracca (CT), 1700 m, 1 ㅇ 8.VIII.1996 (UCTC).

Distribution. Palaearctic region, known from Italy (Scaramozzino, 1995), new for Sicily.

Ichneumon minutorius Desvignes, 1856

Material. Etna, Linguaglossa, Mt. Baracca (CT), $1700 \mathrm{~m}, 1$ ㅇ 7.VII.1995 (UCTC).

Distribution. Palaearctic region, known from Italy (Scaramozzino, 1995), new for Sicily.

\section{Ichneumon languidus Wesmael, 1845}

Material. Etna, Maletto, Contrada Fontanamurata (CT), 1000 m, 1 ○े 7.VI.1998 (UCTC).

Distribution. Palaearctic region, known from Italy (Scaramozzino, 1995), new for Sicily.

Ichneumon quaesitorius Linnaeus, 1761

MATERIAl. Nebrodi, Cesarò, Biviere di Cesarò (ME),
1274 m, 1 ○ 23.VI.1999 (UCTC); Etna, Maletto Contrada Fontanamurata, 1000 m, 1 के 8.X.1995 (UCTC). Distribution. Palaearctic region, known from Italy (Scaramozzino, 1995), new for Sicily.

Ichneumon sarcitorius Linnaeus, 1758

Material. Etna, Mt. Maletto (CT), $1550 \mathrm{~m}, 1$ ○ 24.VII.1992 (STC); Etna, Ragalna, Fossa della Nave (CT), 1700 m, 1 § 10.VIII.2000 (STC); Etna, Maletto, Contrada Fontanamurata (CT), $1000 \mathrm{~m}, 1$ ठ 9.VII.1995; 1 क 1 ठ 29. VI.1997; 1 ○े 28.VI.1998 (UCTC); Etna, Mt. S. Leo (CT), 1000 m, 1 ㅇ 7.VII.1992 (UCTC); Piana di Catania, Contrada Primosole, 1 ô 11.VI.1996; 1 ô $2 . V .1998 ; 1$ त 21.VI.1998 (UCTC).

Distribution. Palaearctic region, known from Italy (Pagliano, 2009) and Sicily (De Stefani Perez, 1895).

\section{Ichneumon tuberculipes Wesmael, 1848}

Material. Nebrodi, Bosco di Malabotta (ME), 1 ㅇ 15.XI.1992 (UCTC); Etna, Milo (CT), 2 ठ 21.VII.1998 (UCTC); Etna, Ragalna, Mt. Vetore (CT), 1700 m, 1 ð 31.VIII.1997 (UCTC); Etna, Castiglione di Sicilia, Foresta Ragabo (CT), 1500 m, 1 우 20.XII.1997; 1 ㅇ 20.XII.1997, under bark of Pinus laricio Poiret, Bella leg. (UCTC); Etna, Maletto, Contrada Fontanamurata, $1000 \mathrm{~m}, 1$ क 9.VII.1995 (UCTC); Etna, Milo (CT), 750 m, 1 ô 21.VII.1998 (UCTC).

Distribution. Palaearctic region, known from Italy (Scaramozzino, 1995), new for Sicily.

TAXONOMICAL REMARK. of the two $\widehat{\partial}$ one has the typical ivory coloration (see Hilpert, 1992), the other one has a small yellowish spot on the scutellum, the body is otherwise completely black.

\section{Ichneumon xanthorius Forster, 1771}

Material. Etna, Zafferana Etnea, Valle San Giacomo (CT), 800 m, 1 ô 25.VI.1995 (UCTC).

Distribution. Palaearctic region, known from Italy (Pesarini, 2009) and Sicily (Scaramozzino, 1995).

\section{Pseudoamblyteles homocerus (Wesmael, 1854)}

Material. Etna Sud, Nicolosi, around Mt. Serra Pizzuta (CT), 950 m, 1 ๆ 30.V.2001 (STC); Etna, Belpasso, Mt. S Leo (CT), 1000 m, 1 đ 7.VI.1992 (STC); 1 ô 22.VI.1991; 1 ô 24.VI.1992; 1 ô 4.VII.1992; 1 
đ̛ 9.VII.1992; 1 đ̊ 2.VI.1993 (UCTC); Etna, San Gregorio $300 \mathrm{~m}, 2$ त and 3 ô 5.V.1994, 1 क 7.V.1995 (UCTC); Etna, Maletto, Contrada Fontanamurata (CT), $1000 \mathrm{~m}, 1$ 우 and $1 \hat{\gamma}$ 9.VII.1995, 1 q 28.VI.1998 (UCTC); Etna, Linguaglossa, Mt. Baracca (CT), 1700 m, 2 ๙ 26. VIII.1994, 1 ㅇ 7.VII.1995, 3 ठै 8.VIII.1996, 1 đ 21.VIII.1996 (UCTC); Etna, Tremestieri Etneo (CT), 350 m, 1 đ 21.V.1994, 1 ㅇ 25.III.1995, 1 24.v.1995 (UCTC); Etna, Milo, Casa Paternò (CT), $1300 \mathrm{~m}, 1$ ठิ 23.V.1992, 4 ठ 22.VIII.1996, 1 q 29.VIII.1996 (UCTC); Motta S Anastasia (CT) S.D. 12/II KM 2, 1 ○े 21.V.2000 (STC); Paternò (CT), Contrada Petulenti, $1 \lambda$ 15.V.1993 (STC); Fiume Alcantara, Calatabiano (CT) 4 đิ 4.VI.1999 (UCTC); Nebrodi, Cesarò, Biviere di Cesarò (ME), 1274 m, 3 đ̊ 23.VI.1998 (UCTC); Iblei, Fiume Vizzini, Poggio Morbano (CT), $600 \mathrm{~m}, 1$ \& and 1 § 1.VI.1996 (UCTC); Piana di Catania, Contrada Primosole, 2 o 4.VI.1995, 2 ô and 6 ô 26.V.1996, 1 $q$ and $4 \hat{\sigma}$ 11.VI.1996 (UCTC); Erei, Calascibetta (Enna) 800 m, 1 ㅇ 1.XI.1994 (UCTC).

Distribution. Holarctic region, known from Italy (Pagliano, 2009) and Sicily (Sichel, 1860; Pic, 1899).

Spilothyrateles illuminatorius (Gravenhorst, 1820)

Material. Etna, Ragalna, Serra La Nave 1730 m, 1 q 26.VI.1999 (UCTC); Etna, Ragalna, Milia (CT), 1250 m, 1 đ 4.XI.1992 (STC); Etna, Adrano, Contrada Solicchiata (CT), 700 m, 1 ○ 1.XI.1994 (STC); Piana di Catania, Contrada Primosole, 1 đ 26.V.1996 (UCTC).

Distribution. Palaearctic region, known from Italy (Scaramozzino, 1995), new for Sicily.

TAXONOMICAL REMARK. Clypeus and face centrally yellowish, frontal and facial orbits reddish.

Spilothyrateles nuptatorius (Fabricius, 1793) syn. fabricii (Gravenhorst, 1829)

Material. Etna, Bronte, Contrada Galvarina (CT), 1870 m, 1 đิ 22.VII.1992, 1 ô 23.VII.1992 (STC); Etna, Adrano, Contrada Solicchiata (CT), 1 ठ 1.XI.1994 (STC); Etna, Linguaglossa, Mt. Baracca (CT), 1700 m, 1 §̊ 7.VII.1995 (UCTC).

DistRIBUTION. Western Palaearctic region, known from Italy (Scaramozzino, 1995 as fabricii), new for Sicily.

Stenobarichneumon basiglytus (Kriechbaumer, 1890) Material. Etna, Maletto, Contrada Fontanamurata
(CT), 1000 m, 1 ठ̊ 8.X.1995 (UCTC); Nebrodi, Foresta di Malabotta, Roccella Valdemone (ME), 1000 m, 3 ô 28.VIII.1995 (UCTC).

Distribution. Palaearctic region, known from Italy (Scaramozzino, 1995), new for Sicily.

Thyrateles haereticus (Wesmael, 1854)

Material. Etna, Ragalna, Serra La Nave (CT), 1730 m, 1 đิ 7.IX.1997 (UCTC); Etna, Belpasso, Mt. Manfré (CT), 1400 m, 1 đ 25.VIII.1995 (UCTC); Fiume Alcantara, Calatabiano (CT) 1 ○े 4.VI.1999 (UCTC); Iblei, Noto, La Burritta (SR), 1 ô 23.X.1994 (UCTC).

Distribution. Palaearctic region, known from Italy (Scaramozzino, 1995), new for Sicily.

Tricholabus strigatorius (Gravenhorst, 1829)

Material. Etna, Maletto, Mt. Maletto (CT), $1550 \mathrm{~m}$, 1 q 24.VII.1992 (STC); Etna, Ragalna, Serra La Nave (CT), $1730 \mathrm{~m}, 1$ \& 26.VI.1999 (UCTC).

Distribution. Palaearctic and Oriental regions, known from Italy (Scaramozzino, 1995), new for Sicily.

TAXONOMICAL REMARK. The $q$ from Serra La Nave has black hind legs (except a small subbasal reddish ring of hind tibia).

Triptognathus atripes (Gravenhorst, 1820)

Material. Etna, Belpasso, Mt. S. Leo (CT), 1000 m, 2 ô 7.VII.1992; 1 ㅇ 27.IV.1994 (UCTC); 1 ㅇ 26.VI.1999 (STC); Etna, Ragalna, Milia (CT), 1250 m, 1 ㅇ (STC); 1 7.VI.1992 (UCTC); Etna, Maletto, Contrada Fontanamurata (CT), $1000 \mathrm{~m}, 1$ 우 29.VI.1997 (UCTC).

Distribution. Palaearctic region, known from Italy (Scaramozzino, 1995) and Sicily (Sichel, 1860; De Stefani, 1895).

Triptognathus fumigator (Gravenhorst, 1820)

Material. Etna, Belpasso, Mt. S. Leo (CT), 1000 m, 1 ð 26.VI.1999 (STC); Etna, Maletto, Contrada Fontanamurata (CT), $1000 \mathrm{~m}, 2$ ๙े 4.VII.1992, 2 ठิ 28.VI.1998 (UCTC); Etna, Linguaglossa, Mt. Baracca (CT), 1700 m, 1 ô 24.VII.1995 (UCTC).

Distribution. Palaearctic region, known from Italy (Bauer, 1936) and Sicily (Scaramozzino, 1995). 
Virgichneumon albosignatus (Gravenhorst, 1829)

Material. Etna, Maletto, Contrada Fontanamurata (CT), 1000 m, 1 ㅇ 7.VII.1996 (UCTC); Etna, Milo, Casa Pietracannone (CT), 1100 m, 1 ô 12.VII.1998 (UCTC).

Distribution. Palaearctic region, known from Italy (Scaramozzino, 1995), new for Sicily.

Virgichneumon digrammus (Gravenhorst, 1820)

Material. Etna, Ragalna, Contrada Milia (CT), 1250

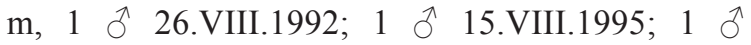
17.VIII.1996 (STC); around Gole dell'Alcantara, 1 우 22.V.1993 (STC); Etna, Ragalna, Mt. Vetore (CT), 1700 m, 1 đै 31.VIII.1997 (UCTC); Etna, Maletto, Contrada Fontanamurata (CT), $1000 \mathrm{~m}, 1 \lambda$ 24.VIII.1995 (UCTC).

Distribution. Palaearctic region, known from Italy (Scaramozzino, 1995), new for Sicily.

Virgichneumon tenuicornis (Heinrich, 1930)

MATERIAL. Etna, Zafferana Etnea, Mt. Zoccolaro (CT) 1600 m, 1 ठ 30.VIII.1995 (UCTC).

Distribution. Known from Southern Europe, new for Italy.

Virgichneumon tergenus (Gravenhorst, 1820)

Material. Madonie, Petralia Sottana, Valle di Zotta Fonda (PA), 1650 m, 1 ठ 14.VII.1993 (UCTC); Etna, Milo, Foresta Cubania (CT), 1400 m, 1 q 7.VIII.1995 (UCTC); Etna, Milo, Casa Paternò (CT), 1300 m , 3 ô 22.VIII.1996 (UCTC); Etna, Milo, Mt. Scorsone (CT), 1600 m, 2 ô 10.VIII.1996 (UCTC); Etna, Castiglione di Sicilia, Caserma Pitarrone (CT), $1450 \mathrm{~m}$, 1 + 30.VII.1998 (UCTC); Etna, Ragalna, Serra La Nave (CT), 1730 m (), 1 ㅇ 7.IX.1997 (UCTC); Etna, Sant'Alfio, Contrada Cerrita (CT), $1400 \mathrm{~m}, 2$ กิ 21.VIII.1998, 1 + 9.V.1999 (UCTC); Etna, Belpasso Mt. Manfrè (CT), 1400 m, 1 q 25.VIII.1995 (UCTC). Distribution. Palaearctic region, known from Sicily (De Stefani Perez, 1895).

Vulgichneumon deceptor (Scopoli, 1763)

Material. Etna, Ragalna, Contrada Milia (CT), 1400 m, 1 đ̊ 10.VIII.1999 (STC); Etna, Ragalna, Mt. Vetore (CT), 1700 m, 1 ઈ 31.VIII.1997 (UCTC); Etna, Bronte, Mt. Palestra (CT), 1900 m, 1 ○े 24.VIII.1996 (UCTC).

Distribution. Palaearctic region, known from Italy (Scaramozzino, 1995), new for Sicily.

Vulgichneumon saturatorius (Linnaeus, 1758)

Material. Etna, Linguaglossa, Mt. Baracca (CT), $1700 \mathrm{~m}, 2$ ○े 29.VII.1998 (UCTC).

Distribution. Palaearctic region, known from Italy (Pagliano, 2009) and Sicily (De Stefani Perez, 1895).

\section{ConClusions}

In total, 79 species of the subfamily Ichneumoninae are recorded, of which 39 are new findings for Sicily, and 9 also to Italy, including a new recently described species of the genus Platylabus. With these new records, the species number of Ichneumoninae presently known for Sicily has been increased up to 105.

The high number of the newly recorded species for Sicily confirms how poor is the knowledge of the Sicilian Ichneumoninae. Upon this consideration, it is not possible for now to provide any biogeographical remarks. Most part of the new recorded species is widespread in the Palaearctic region or its Western part. Anyway, three species: Coelichneumon graecus Horstmann, 2002 and Coelichneumon oltenensis Constantineanu, Pirvescu \& Mihalache, 1979 and Coelichneumon quadriannulatus (Gravenhorst, 1829), all new to Italy, are remarkable with regard to distribution, since previously known from only a few European Countries. Moreover, Platylabus rufator Riedel, 2012 is presently known from only Etna volcano.

Further researches will probably increase the current number of species of Ichneumoninae, especially through better exploration of the montane habitats of Sicily (Madonie, Nebrodi, Peloritani and Etna) and the south-eastern Iblean massif.

\section{ACKNOWLEDGMENTS}

We are grateful to Salvatore Tomarchio for allowing us to study his personal collection. 


\section{REFERENCES}

Alma A. \& Arzone A., 1994 - Notizie fitopatologiche ed epidemiologiche su nottuidi in vigneti piemontesi. Atti XVII Congresso Nazionale italiano di Entomologia, Udine: 559-562.

BAUER E., 1936 - Beitrag zur Ichneumoniden-Fauna von San Valentino alla Muta (Val Venosta). Bollettino della Società Entomologica Italiana 68: 103-111.

CeCConi G., 1924 - Manuale di Entomologia forestale. Padova: 1-680.

De Stefani Perez T., 1882 - Notizie imenotterologiche. Il Naturalista siciliano 2: 55-58.

De Stefani Perez T., 1883 - Miscellanea imenotterologica. Il Naturalista siciliano 2: 280-284.

De Stefani Perez T., 1884 - Imenotteri nuovi o poco conosciuti della Sicilia. Il Naturalista Siciliano 3: 153-158.

De Stefani Perez T., 1885 - Imenotteri nuovi o poco conosciuti della Sicilia. Il Naturalista Siciliano 4: 185-189.

De Stefani Perez T., 1886 - Raccolte imenotterologiche sui Monti di Renda e loro adiacenze. Il Naturalista siciliano 5: 181-186.

De Stefani Perez T., 1887a - Un nuovo genere di Crabronidi ed altri Imenotteri nuovi o poco cogniti raccolti in Sicilia. Il Naturalista Siciliano 6: 85-90.

De Stefani Perez T., 1887b - Due nuovi Ichneumoni di Sicilia. Il Naturalista Siciliano 7: 53-54.

De Stefani Perez T., 1894 - Imenotteri di Sicilia raccolti nel territorio di Santa Ninfa in Provincia di Trapani. Il Naturalista siciliano 13: 199-203; 211-219.

De Stefani Perez T., 1895 - Catalogo degli Imenotteri di Sicilia. Il Naturalista Siciliano 14: 169-182.

HorSTMANN K., 1993 - Revision der von Ferdinand Rudow beschriebenen Ichneumonidae I. Beitr Ent 43: 3-38.

HoRstMAnN K., 2002 - Über einige mit Coelichneumon orbitator (Thunberg, 1824) nah verwandte Arten (Hymenoptera, Ichneumonidae, Ichneumoninae). Entomofauna 23: 73-84.

HoRSTMANN K., 2007 - Revision der westpaläarktischen Arten von Ansiobas Wesmael, 1845 (Hymenoptera, Ichneumonidae, Ichneumoninae). Entomofauna 28: 93-116.

Pagliano G., 2003 - Ricerche imenotterologiche nelle isole di Lampedusa e Pantelleria. Il Naturalista siciliano 27 (1-2): 115-149.

Pagliano G., 2009 - Le collezioni del Museo Regionale di Scienze Naturali di Torino. Ichneumonidae della fauna d'Italia e citazioni di nuove specie per il Piemonte (Hymenoptera). Bollettino del Museo regionale di Scienze Naturali di Torino 27: 153-240.

Pagliano G., Scaramozzino P.L., 1995 - Hymenoptera Gasteruptionidae, Ichneumonidae e Aculeata (esclusi Chrysidoidea, Mutillidae e Formicidae, in Massa B. (ed.), Arthropoda di Lampedusa, Linosa e Pantelleria (Canale di Sicilia, Mar Mediterraneo). Il Naturalista siciliano, 19: 723-738.

Pesarini F., 2009 - Contributo preliminare sugli Imenotteri Terebranti (non Evanioidei) della collezione Campadelli. VII contributo. Annali del Museo Civico di Storia Naturale di Ferrara 11: 1-115.

PIC M.T., 1899 - Sur quelques Ichneumoniens de la Coll. Tournier. Revue Scientifique du Bourbonnais 12: 100-103.

Riedel M., 2008 - Revision der westpaläarktischen Platylabini: 1. Die Gattung Platylabus Wesmael, 1845 (Hymenoptera, Ichneumonidae, Ichneumoninae). Spixiana 31: 105-172.

Riedel M., Tomarchio S., 2012a - A contribution to the Ichneumoninae fauna of Sicily (Hymenoptera Ichneumonidae). Bollettino della Società entomologica italiana 144 (3): 125-135.

Riedel M., Tomarchio S., 2012b - Retraction - A contribution to the Ichneumoninae fauna of Sicily (Hymenoptera Ichneumonidae). Bollettino della Società entomologica italiana 144 (3): a.

Riggio G., De Stefani-Perez T., 1888 - Sopra alcuni Imenotteri dell'Isola di Ustica. Il Naturalista Siciliano 7: 145-150.

Roberti D., Frilli F., PizZaghi W., 1965 - Contributo alla conoscenza dell'entomofauna del Piacentino (Specie raccolte nel decennio 1955-1964). Entomologica 1: 1-118.

Rudow F., 1888 - Einige neue Ichneumoniden. Entomologische Nachrichten 14 (9): 129-136.

Scaramozzino P.L., 1995 - Hymenoptera Ichneumonidae. In: Minelli, A., Ruffo, S. \& La Posta S. (eds.), Checklist delle specie della fauna italiana, 94. Calderini, Bologna.

Sichel J., 1860 - Liste des Hymenopteres recueilis en Sicile par Mt. E. Bellier de la Chavignerie pendant les mois d'Aout a Septembre 1859. Annales de la Societé Entomologique de France (3) 8: 749-764.

TURrisi G.F., SchÖNITZER K., Diller E., 2007 - Ichneumon flies from Sicily, with descriptions of new taxa (Hymenoptera, Ichneumonidae: Ichneumoninae: Phaeogenini and Diplazontinae). Mitteilungen der Münchner Entomologische Gesellschaft 97: 115-126.

Yu D.S., HoRstMAnN K., 1997 - Catalogue of world Ichneumonidae (Hymenoptera). Mem. American Ent. Inst. 58 : 1-1558.

Yu D.S., van AChterberg K., Horstmann K., 2005 - World Ichneumonidea 2004. Taxonomy, Biology, Morphology and Distribution. CD/DVD. Taxapad, Vancouver, Canada.

Authors' address:

Matthias Riedel, Amselweg 9 A, 29683 Bad Fallingbostel, Germany. E-mail: mamaflo.riedel@t-online.de Giuseppe Fabrizio Turrisi, via Cristoforo Colombo 8, 95030 Pedara, Italy. E-mail: turrisifabrizio@yahoo.it 